\title{
Closed-form, robust and accurate multi-frequency phase unwrapping: frequency design and algorithm ${ }^{\text {光 }}$
}

\author{
Li Wei, Wangdong Qi* \\ PLA University of Science and Technology, Nanjing, China
}

\begin{abstract}
A closed-form algorithm, named "concerto", is proposed for phase-based distance estimation in multi-frequency phase unwrapping (MFPU) system. The concerto method consists of three coherent estimation stages,i.e., initial modified BW estimation, residual error estimation and LS estimation, each of which has a closed-form expression and cooperates closely with each other like a concerto. Due to a specially designed frequency pattern, concerto is reliable, accurate, and computationally simple. Meanwhile, measurement frequency selection is an easier task. Performance comparisons with beat wavelength (BW), excess fractions (EF) and Chinese remainder theorem (CRT) method confirm that our method outperforms these methods both in accuracy and reliability and can asymptotically achieve the Cramér-Rao bound (CRB).
\end{abstract}

Keywords: Multi-frequency phase unwrapping (MFPU), synthetic aperture radar (SAR), Chinese remainder theorem (CRT), beat wavelength (BW), frequency pattern, real-time, closed-form

\section{Introduction}

Precise distance or height measurement is of great importance for many fields such as geodesy [1, 2], synthetic aperture radar (SAR) [3, 4] or interferometric synthetic aperture radar (InSAR) [8] and optics [11, 10]. In these cases, phase measurements at multiple frequencies are used for accurate estimation of distance or height. The obstacle met in MFPU is that the measured phases are wrapped into the range $(-\pi, \pi]$, while the true distance is related to the unwrapped phases. To recover the unwrapped phases, unknown integer called folding integer must be determined by a phase unwrapping method.

A least-square grid search is used to solve the phase unwrapping problem in [1]. Since the processing time is dependent on both the range and the search step, it is usually computationally prohibitive for real-time applications. If the measurement wavelengths can be scaled to integers and these integers are pairwise co-prime, the CRT algorithm may be applied[3, 4, 5, 2]. Although the traditional CRT is computationally attractive owing to its closed-form solution, it is very sensitive to phase noise[7]. More importantly, the frequency selection is a challenge since the co-prime condition must be met for any pair of frequencies [2]. In order to solve the noise sensitivity problem, a two-dimensional searching based robust CRT has been proposed in [3]. Thus, there exists a compromise between reliability and complexity. The two-dimensional searching used in [3] is later reduced to one-dimensional searching by [4]. To further reduce the computational complexity of searching based CRT, closed-form and robust CRT are presented in [5, 8]. Recently, new closed-form phase unwrapping using lattice theory is proposed in [9], which has similar complexity and accuracy as the CRT in [5]. But the rigid requirement on measurement frequencies still exists [5, 9]. The robust CRT proposed in [2] both alleviates the requirement on frequencies and improves the resistance to noise, at the cost of very limited measurement range. The classical EF method has also been proposed for decades, see

\footnotetext{
This work has been supported by the National Natural Science Foundation of China (No.61402520; No.61273047; No.61573376) and the Natural Science Foundation of Jiangsu Province (BK20130068)

${ }^{*}$ Corresponding author

Email addresses: wlnb@hotmail.com (Li Wei ), wangdongqi@gmail.com ( Wangdong Qi )
} 
[10] and references therein, which searches all the possible locations determined at the shortest wavelength. At the most likely location, all folding integers calculated are closest to integer simultaneously[10]. Because the processing time is increased linearly with range, EF approach is still computationally intensive. Compared with EF, the popular BW method has similar reliability, yet requires very low computation cost[11].

However, BW estimates the folding integer one by one and leads to accuracy loss since only partial information is used for each folding integer estimation. The frequency pattern designed for BW may cause poor estimation performance in case of large number of frequencies[11, 18]. In this letter, another closed-form phase unwrapping method, named concerto, is provided.

The main features of this algorithm include:

i) Large (Adjustable) measurable range, low computation complexity, high reliability and accuracy can be achieved simultaneously.

ii) Frequency selection is easier than CRT based method.

\section{Signal model and BW method}

Consider a multi-frequency ranging system using phase measurements recorded at multiple wavelengths [2, 9]. Assume the wrapped phase (or principal phase) measurements at $N$ wavelengths $\lambda_{0}<\lambda_{1} \cdots<\lambda_{N-1}$ are $\phi_{0} \cdots \phi_{N-1}$. In MFPU, the ideal noise-free measurement phases are related to the range $L$ by 1

$$
\phi_{i}=\left[2 \pi \frac{L}{\lambda_{i}}\right]_{2 \pi}, 0 \leq i \leq N-1
$$

In BW method, using the general formula

$$
\left[[x]_{2 \pi}-[y]_{2 \pi}\right]_{2 \pi}=[x-y]_{2 \pi}
$$

the beat (synthetic) phases $\Phi_{i}$ and beat wavelengths $\Lambda_{i}$ can be formed as

$$
\begin{aligned}
\Phi_{i} & =\left[\phi_{0}-\phi_{i}\right]_{2 \pi}=\left[2 \pi \frac{L}{\lambda_{0}}-2 \pi \frac{L}{\lambda_{i}}\right]_{2 \pi}=\left[2 \pi \frac{L}{\Lambda}\right]_{2 \pi} \\
\Lambda_{i} & =\lambda_{i} \lambda_{0} /\left(\lambda_{i}-\lambda_{0}\right)=c /\left(f_{0}-f_{i}\right), \quad 1 \leq i \leq N-1
\end{aligned}
$$

where $\lambda_{i}=c / f_{i}$ and $c$ is the speed of light, $[\cdot]_{2 \pi}$ denotes a modulo- $2 \pi$ operation. Equation (1) is equivalent to

$$
L=\left(m_{i}+\phi_{i} / 2 \pi\right) \lambda_{i}, \quad 0 \leq i \leq N-1
$$

and (2) can be expressed as

$$
\begin{aligned}
L & =\left(M_{i}+\Phi_{i} / 2 \pi\right) \Lambda_{i} \\
& =\left(M_{i+1}+\Phi_{i+1} / 2 \pi\right) \Lambda_{i+1}, \quad 1 \leq i \leq N-2
\end{aligned}
$$

where $m_{i}, M_{i}$ are the folding integers at wavelength $\lambda_{i}$ and beat wavelength $\Lambda_{i}$ respectively. Note that the unambiguous measurement range (UMR) is equal to the largest beat wavelength $\Lambda_{1}$ in BW method and the unknown range $L$ will satisfy $|L|<\Lambda_{1} / 2=\frac{c}{2\left(f_{0}-f_{1}\right)}$ by proper choice of $\Lambda_{1}$. The same assumption is made in this letter. Then we have $M_{1}=0$ immediately. Now, consider the realistic measurement phases with phase noise $\theta_{e}(i)$,

$$
\phi_{i}=\left[2 \pi \frac{L}{\lambda_{i}}+\theta_{e}(i)\right]_{2 \pi}, 0 \leq i \leq N-1
$$

\footnotetext{
${ }^{1}$ Note that similar problems and signal model arise in optic [11], SAR [4], InSAR imaging system [8], frequency estimation [6] and single source direction of arrival (DOA) estimation[17].
} 
using (4) and (5), the integers $M_{i}, m_{0}$ can be calculated sequentially as follows:

$$
\begin{aligned}
M_{i+1} & =\operatorname{round}\left[\left(M_{i}+\frac{\Phi_{i}}{2 \pi}\right) \frac{\Lambda_{i}}{\Lambda_{i+1}}-\frac{\Phi_{i+1}}{2 \pi}\right] \\
m_{0} & =\operatorname{round}\left[\left(M_{N-1}+\frac{\Phi_{N-1}}{2 \pi}\right) \frac{\Lambda_{N-1}}{\lambda_{0}}-\frac{\phi_{0}}{2 \pi}\right]
\end{aligned}
$$

where round[·] denotes rounding to the nearest integer. It follows that

$$
L=\left(m_{0}+\phi_{0} / 2 \pi\right) \lambda_{0}
$$

\section{Frequency design}

Suppose the phase noise $\theta_{e}(i)$ at each wavelength $\lambda_{i}$ is zero-mean Gaussian noise with identical standard deviation $\sigma$, then the error in (7), before the rounding operation, is also zero-mean Gaussian noise with deviation $\sigma_{e} 2$,

$$
\sigma_{e}=\frac{\sqrt{2} \sigma}{2 \pi} \sqrt{\left(\frac{\Lambda_{i}}{\Lambda_{i+1}}\right)^{2}+1}
$$

According to [11], to maximize noise immunity, the phase noise introduced in each $M_{i}$ estimation of (7) should have identical standard deviation, and thus the scaling factor $\Lambda_{i} / \Lambda_{i+1}$ must be all equal to each other 3 .

Therefore, the frequency pattern designed for BW method is [11]

$$
\frac{\Lambda_{1}}{\Lambda_{2}}=\frac{\Lambda_{2}}{\Lambda_{3}}=\cdots=\frac{\Lambda_{N-1}}{\lambda_{0}}
$$

or equivalently,

$$
\frac{f_{0}-f_{2}}{f_{0}-f_{1}}=\frac{f_{0}-f_{3}}{f_{0}-f_{2}}=\cdots=\frac{f_{0}}{B}
$$

where $B=f_{0}-f_{N-1}$ denotes the measurement bandwidth.

Note that the last equality in (12) is needed to unwrap $m_{0}$ using (8) since it is the final step in the estimation chain. The final absolute error will be smaller than $\lambda_{0} / 2$ when $m_{0}$ has been correctly unwrapped. However, the last step of calculating $m_{0}$ in $(8)$ is not essential if the method to be described in the next section is used. Then, the constraint of $\Lambda_{i} / \Lambda_{i+1}=\frac{f_{0}}{B}$ could be removed. This is a key step to achieve high-accuracy estimation. Since $\frac{f_{0}}{B}$ is usually large, the measurement frequencies will converge quickly to $f_{0}$ with increased frequency number. This kind of frequency pattern will lead to poor estimation accuracy, see [18] for detail. The proposed frequency pattern used in concerto, takes the form

$$
r=\frac{f_{0}-f_{2}}{f_{0}-f_{1}}=\frac{f_{0}-f_{3}}{f_{0}-f_{2}}=\cdots=\frac{f_{0}-f_{N-1}}{f_{0}-f_{N-2}}
$$

multiplying the last $N-1-i$ terms of (13)

$$
f_{i}=f_{0}-\left(f_{0}-f_{N-1}\right) r^{-(N-1-i)}, i=1,2, \cdots, N-2
$$

\footnotetext{
${ }^{2}$ There may be other phase error, i.e. due to multipath, which we could cancel by antenna design or multi-frequency average[19], this is out of the scope of the paper.

${ }^{3}$ This principle can be interpreted using the "Barrel Theory", which states that the capacity of a barrel is limited by the shortest stave. Therefore, if the $M_{i}$ estimation has been corrupted by the maximal phase noise, then it is "the shortest stave" and is prone to error. When the error happens, it propagates along the estimation chain in 7 .
} 
where $f_{0}$ and $f_{N-1}$ are the pre-determined highest and lowest frequency. Suppose $|L|<K / 2, K$ is the maximum measurable range of ranging system. Since we require $U M R \geq K$ with $U M R=\Lambda_{1}=\frac{c}{f_{0}-f_{1}}$, the ratio $r$ is adjusted adaptively according to the frequency number $N$,

$$
\begin{gathered}
r^{N-2}=\frac{f_{0}-f_{N-1}}{f_{0}-f_{1}}=\frac{B \cdot U M R}{c} \geq \frac{B \cdot K}{c} \\
r \geq \sqrt[N-2]{\frac{B \cdot K}{c}}
\end{gathered}
$$

We set $r=\sqrt[N-2]{B \cdot K / c}$ for maximizing the tolerance of noise, see (10). Frequency pattern design is then easily accomplished by simply setting $r$ in concerto.

Remark 1: Under the constraint of being larger than unity, the ratios in (13) are required to be not only equal, but also as small as possible. Since $U M R=\frac{B}{c} r^{N-2}$, even a ratio slightly greater than unity will produce an extremely large $U M R$ thanks to the exponent increase property. Error accumulation is avoided by decision at each step of (7).

\section{Proposed Method}

In this section, we present the concerto method which adds two optimal estimations to a modified BW method (using the proposed frequency pattern) and achieves high accuracy with extreme low computation cost due to closedform solution developed. The modified BW method still suffers from accuracy loss since only partial information is exploited in each phase unwrapping stage using just two synthetic wavelengths, see (7). With two additional steps, concerto aims to recover the lost information by making full use of all the phase information.

We use (7) for a rough estimation of $L$ in the first of three stages. The estimation error of the last step in (7) lies in $\left[-c / 2\left(f_{0}-f_{N-1}\right), c / 2\left(f_{0}-f_{N-1}\right)\right]=[-c /(2 B), c /(2 B)]$, provided that $M_{N-1}$ has been correctly estimated. Then a coarse estimate $L_{c}$ is

$$
L_{c}=M_{N-1} \Lambda_{N-1}+\frac{\Phi_{N-1}}{2 \pi} \Lambda_{N-1}
$$

The original wrapped phase $\phi_{i}$ will be compensated as follows

$$
\tilde{\phi}_{i}=\left[\phi_{i}-\frac{2 \pi L_{c}}{\lambda_{i}}\right]_{2 \pi}=\left[\frac{2 \pi\left(L-L_{c}\right)}{\lambda_{i}}+\theta_{e}(i)\right]_{2 \pi}
$$

Denote the residual error $L_{r}=L-L_{c}$. To obtain $L_{r}$, we construct the following cost function

$$
\begin{aligned}
& \max _{\hat{L}_{r}}\left|\sum_{i=0}^{N-1} \exp \left\{j\left(\frac{2 \pi \hat{L}_{r}}{\lambda_{i}}-\tilde{\phi}_{i}\right)\right\}\right|^{2} \\
& =\max _{\hat{L}_{r}}\left|\sum_{i=0}^{N-1} \exp \left\{j\left(\frac{2 \pi f_{i} \hat{L}_{r}}{c}-\tilde{\phi}_{i}\right)\right\}\right|^{2}
\end{aligned}
$$

where $\hat{L}_{r}$ is the estimation of $L_{r}$. This is because when $\hat{L}_{r}=L_{r}$, all the unit-vector $e^{j\left(2 \pi f_{i} \hat{L}_{r} / c-\tilde{\phi}_{i}\right)}, i=0, \cdots, N-1$, will be aligned to the same direction and the cost function will achieve its maximum value.

Let $L_{r}^{*}$ be the optimal estimation of $L_{r}$. When $\left|L_{r}\right|<c / 2 B$, $L_{r}^{*}$ must be of the form (see Appendix A)

$$
L_{r}^{*}=\frac{c}{2 \pi} \frac{\Delta \mathbf{f}^{T} \boldsymbol{\Gamma}^{T} \mathbf{R}^{-1} \boldsymbol{\Gamma} \Delta \tilde{\mathbf{\Phi}}}{\Delta \mathbf{f}^{T} \boldsymbol{\Gamma}^{T} \mathbf{R}^{-1} \boldsymbol{\Gamma} \Delta \mathbf{f}}
$$

\footnotetext{
${ }^{4}$ Eq. 19 does not hold true for $\left|L_{r}\right|>c / 2 B$, see Appendix Appendix A for details. Therefore, $\left|L_{r}\right|<c / 2 B$, guaranteed by the first stage of concerto, forms the core of the second stage.
} 
where $\Delta \mathbf{f}=\left[\Delta f_{1}, \Delta f_{2}, \cdots \Delta f_{N-1}\right]^{T}, \Delta f_{i}=f_{i-1}-f_{i}, \Delta \tilde{\mathbf{\Phi}}=\left[\Delta \tilde{\phi}_{0,1}, \Delta \tilde{\phi}_{1,2}, \cdots, \Delta \tilde{\phi}_{N-2, N-1}\right], \Delta \tilde{\phi}_{i, k}=\left[\tilde{\phi}_{i}-\tilde{\phi}_{k}\right]_{2 \pi}$, and $\mathbf{R}^{-1}$, $\boldsymbol{\Gamma}$ are defined as

$$
\begin{gathered}
\boldsymbol{\Gamma}=\left[\begin{array}{cccc}
1 & 0 & \cdots & 0 \\
1 & 1 & \cdots & 0 \\
\vdots & \vdots & 1 & 0 \\
1 & 1 & 1 & 1
\end{array}\right] \\
\mathbf{R}^{-1}=\mathbf{I}_{N-1}-\frac{\mathbf{u u}^{T}}{N}, \mathbf{u}=[1,1, \cdots 1]^{T}
\end{gathered}
$$

where $\mathbf{I}_{N}$ denotes a $N \times N$ identity matrix. Let $\mathbf{W}=\Gamma^{T} \mathbf{R}^{-1} \boldsymbol{\Gamma}$, which is a constant, with the $(j, k)$ entry $\mathbf{W}_{j k}$,

$$
\mathbf{W}_{j k}=\frac{N \min (j, k)-j k}{N}, 1 \leq j, k \leq N-1
$$

Then, (19) can be simplified as

$$
L_{r}^{*}=\frac{c}{2 \pi} \frac{\Delta \mathbf{f}^{T} \mathbf{W} \Delta \tilde{\boldsymbol{\Phi}}}{\Delta \mathbf{f}^{T} \mathbf{W} \Delta \mathbf{f}}
$$

The optimal estimation of the second stage is readily obtained

$$
L_{m}=L_{r}^{*}+L_{c}
$$

If the residual error $\left|L-L_{m}\right|$ of (22) is small enough, and the folding integer vector $\hat{\mathbf{m}}=\left[\hat{m}_{0}, \hat{m}_{1}, \cdots, \hat{m}_{N-1}\right]$ could be estimated correctly by

$$
\hat{m}_{i}=\operatorname{round}\left[\frac{L_{m}}{\lambda_{i}}-\frac{\phi_{i}}{2 \pi}\right]
$$

Then the least square (LS) based cost function in MFPU could be written as

$$
\begin{aligned}
J(L) & =\sum_{i=0}^{N-1}\left(\left[\frac{2 \pi L}{\lambda_{i}}-\phi_{i}\right]_{2 \pi}\right)^{2} \\
& =\sum_{i=0}^{N-1}\left(\frac{2 \pi L}{\lambda_{i}}-\phi_{i}-2 \pi \hat{m}_{i}\right)^{2}
\end{aligned}
$$

Denote $\Lambda_{i n v}=\left[\frac{1}{\lambda_{0}}, \frac{1}{\lambda_{1}}, \cdots, \frac{1}{\lambda_{N-1}}\right]$ and $\mathbf{m}_{\mathbf{f}}=\left[m_{f 0}, m_{f 1}, \cdots, m_{f N-1}\right], m_{f i}=\hat{m}_{i}+\frac{\phi_{i}}{2 \pi}$, then

$$
J(L)=4 \pi^{2}\left(\Lambda_{i n v} L-\mathbf{m}_{\mathbf{f}}\right)\left(\Lambda_{i n v} L-\mathbf{m}_{\mathbf{f}}\right)^{T}
$$

The optimal estimation $L^{*}$ of concerto that minimizing $J(L)$ is finally obtained

$$
L^{*}=\frac{\Lambda_{i n v} \mathbf{m}_{\mathbf{f}}^{T}}{\Lambda_{i n v} \Lambda_{i n v}^{T}}
$$

It is easy to verify that

$$
\begin{aligned}
& \Delta L=L^{*}-L=\frac{\sum_{k=0}^{N-1} \lambda_{k}^{-1} \theta_{e}(k)}{2 \pi \sum_{k=0}^{N-1} \lambda_{k}^{-2}} \\
& \mathbf{E}[\Delta L]=0 \\
& \mathbf{E}\left[(\Delta L)^{2}\right]=\frac{1}{4 \pi^{2}} \frac{\mathbf{E}\left[\left(\sum_{k=0}^{N-1} \lambda_{k}^{-1} \theta_{e}(k)\right)^{2}\right]}{\left(\sum_{k=0}^{N-1} \lambda_{k}^{-2}\right)^{2}} \\
& =\frac{\sigma^{2}}{4 \pi^{2} \sum_{k=0}^{N-1} \lambda_{k}^{-2}}
\end{aligned}
$$


Table 1: Computation cost of the EF, BW, DCRT and concerto method using the frequency pattern described below (both $K=144 \mathrm{~m}$ and $K=14400 \mathrm{~m}$ are evaluated )

\begin{tabular}{|l|c|c|c|c|}
\hline Method & $\begin{array}{c}\text { Range } \\
(\mathrm{m})\end{array}$ & $\begin{array}{c}\text { Process Time } \\
(\mathrm{ms})\end{array}$ & $\begin{array}{c}\text { Range } \\
(\mathrm{m})\end{array}$ & $\begin{array}{c}\text { Process Time } \\
(\mathrm{ms})\end{array}$ \\
\hline EF & 144 & 7.62 & 14400 & 862.15 \\
\hline BW & 144 & 0.11 & 14400 & 0.12 \\
\hline DCRT & 144 & 3.59 & 14400 & N/A \\
\hline concerto & 144 & 0.21 & 14400 & 0.23 \\
\hline
\end{tabular}

where $\mathbf{E}[\cdot]$ is the expectation operation and $\mathbf{E}\left[\theta_{e}(k) \theta_{e}(j)\right]=0$ for $k \neq j$.

Remark 2: Note that no matrix inversion or matrix decomposition is required in the computation of $L^{*}$. So, concerto is suitable for hardware implementation.

The Cramer-Rao bound (CRB) of $L$ is also shown for comparison (see Appendix B)

$$
\operatorname{CRB}(L)=\frac{\sigma^{2}}{4 \pi^{2}}\left(\sum_{k=1}^{N} \lambda_{k}^{-2}\right)^{-1}
$$

From Eqs. (29) and (30), it is worth noting that the estimator given by Eqs. (16)-(26) has attained the optimal accuracy by utilizing all the phase information simultaneously.

\section{Simulation Analysis}

Unless otherwise mentioned, simulations are performed under the following conditions: For fair comparison of the robust dual-frequency CRT (DCRT) [2], EF, BW and concerto method, simulations are compared under the same bandwidth and frequency number. The highest and lowest frequency are set as $f_{0}=2500 \mathrm{MHz}, f_{N-1}=2400 \mathrm{MHz}$, with $B=100 \mathrm{MHz}$, and $N=51$. For DCRT method, the frequency pattern is designed as in [2] with quantization step $u=0.0001 \mathrm{~m}, C=1$ and $R=30 \mathrm{~m}$. For the other methods, the proposed frequency pattern is used with $K=144 \mathrm{~m}$ (the same as that in [2]) and $r=\sqrt[40]{B \cdot K / c}$. SNR is defined as $\operatorname{SNR}=\frac{1}{2 \mathbf{E}\left[\theta_{e}^{2}(k)\right]}=\frac{1}{2 \sigma^{2}}$.

In Table 11 the relative computational load is represented by process time collected in Matlab, running on a $2.33 \mathrm{GHz}$ processor with $2 \mathrm{~GB}$ memory. The $\mathrm{BW}$ and concerto method have comparable complexity, which both are almost independent of range and lower than DCRT or EF method. As expected, the computation cost of EF is much higher than the others and linearly increased with range. Note that the process time of DCRT is not simulated for larger range because of its limited measurement range.

The mean square error (MSE) of different methods, defined as $M S E(L)=\mathbf{E}\left[(\hat{L}-L)^{2}\right]=\mathbf{E}\left[(\Delta L)^{2}\right]$, where $\hat{L}$ is the estimated value of the true $L$, are shown in Fig 1 It reveals that concerto remarkably outperforms the others in MSE performance for the whole SNR region simulated. The CRB for the frequency pattern in [2] and the proposed frequency pattern are also shown (denoted as CRB-DCRT and CRB-proposed respectively). Both CRB are observed to be almost completely overlapped. Furthermore, only the proposed approach asymptotically attains the CRB for high SNR, thus possessing the optimal accuracy and validating the theoretical analysis in (29).

The probability $P\left(|\Delta L|>\lambda_{0}\right)$ is of particular interest for accurate ranging. In Fig 2 the reliability of concerto, represented by the probability $P\left(|\Delta L|>\lambda_{0}\right)$, is far more superior to the others. For example, compared with the others, about $3 \mathrm{~dB}$ to even $10 \mathrm{~dB}$ gain is obtained for concerto under the same reliability. It is interesting to note that the robust DCRT method has poor MSE performance but with relatively better reliability.

In Fig 3, we compare the reliability $\left(P\left(|\Delta L|>\lambda_{0}\right)\right)$ of three closed-form method (the concerto and two CRTbased estimator, i.e. DCRT and the closed-form CRT (CCRT) [5]), in case of small number of frequencies, i.e. $N=4, N=6$ and $N=8$. Under the same bandwidth and frequency number, two set of frequency patterns (or wavelength patterns) are used to meet the requirement of each estimator. The proposed frequency pattern is used with $K=10000 \mathrm{~m}$ for concerto. As a result, the wavelengths used for concerto are $\lambda=\{1.1,1.1001,1.1075,1.9\}$, 


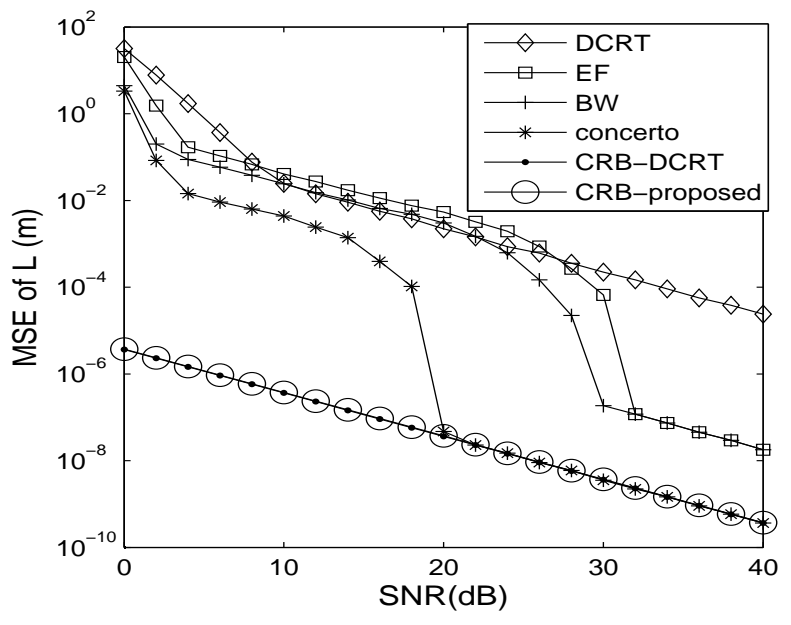

Figure 1: MSE versus SNR for different methods with $N=51$ and $B=100 \mathrm{MHz}$.

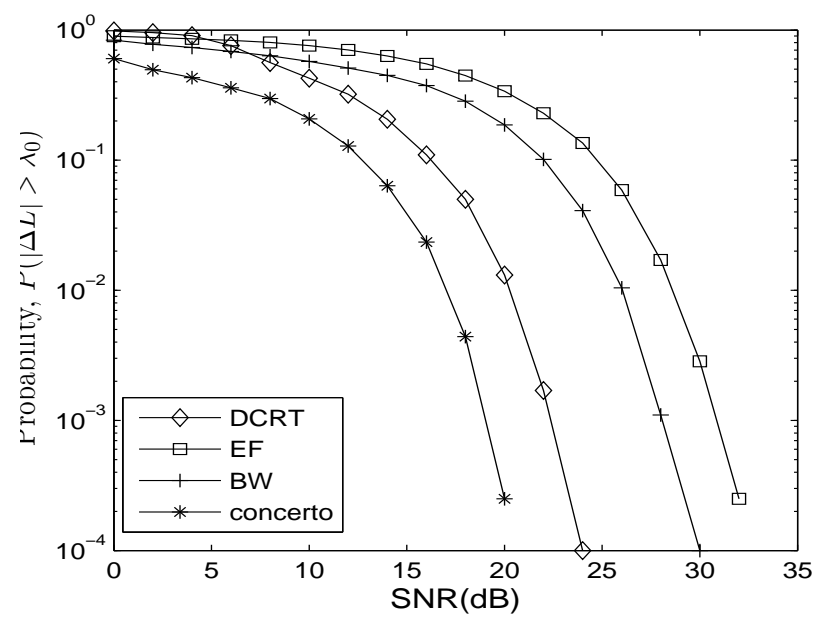

Figure 2: Probability of the absolute error larger than the wavelength $\lambda_{0}$ with $N=51$ and $B=100 \mathrm{MHz}$.

$\lambda=\{1.1,1.1001,1.1011,1.1092,1.1849,2.9\}$, and $\lambda=\{1.1,1.1001,1.1005,1.1023,1.1098,1.1433,1.3144,3.7\}$ for $N=4, N=6$ and $N=8$. Correspondingly, the wavelengths patterns used for both DCRT and CCRT are $\lambda=\{1.1,1.3,1.7,1.9\}, \lambda=\{1.1,1.3,1.7,1.9,2.3,2.9\}, \lambda=\{1.1,1.3,1.7,1.9,2.3,2.9,3.1,3.7\}$. The reliability of concerto is dramatically improved with frequency number $N$. On the contrary, the reliability of both DCRT and CCRT are reduced rather than improved when frequency number is increased, with DCRT slightly outperforming CCRT. Therefore, DCRT and CCRT are more suitable for the case that the useable frequencies are very limited while concerto benefits from an increase of frequency number. Moreover, concerto is superior to the others even for relatively small number of frequencies, i.e. $N \geq 6$.

The assumption that the estimation error $L_{r}$ of the first stage of concerto satisfies $\left|L_{r}\right|<c / 2 B$, plays a great role in concerto. To validate this hypothesis, Fig 4 shows the impact of measurement range $K$ on the probability $P\left(\left|L_{r}\right|>c / 2 B\right)$ for relatively low $\mathrm{SNR}=5 \mathrm{~dB}$. With $N=16$, the probability is observed to remain small even for $K$ as large as $100 \mathrm{~km}$. In other words, with mild assumptions on SNR and $N$, the measurement range of concerto may be greatly extended by increasing $K$ while maintaining a very low probability $P\left(\left|L_{r}\right|>c / 2 B\right)$.

In Fig 5, with a fixed $K=100 \mathrm{~km}$, we investigate the impact of frequency number $N$ on SNR threshold of concerto. 


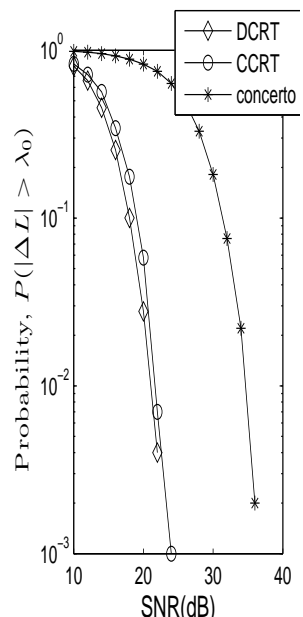

(a) Case I

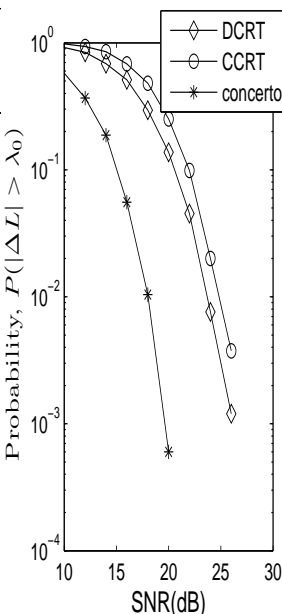

(b) Case II

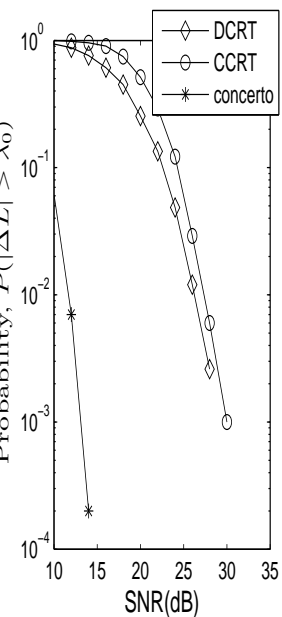

(c) Case III

Figure 3: Probability of the absolute error larger than the wavelength $\lambda_{0}$ with varying N. (a) $N=4$. (b) $N=6$. (c) $N=8$.

If SNR is below the threshold, the estimation accuracy will deteriorate significantly and the result becomes completely useless 5 . Therefore, the SNR threshold should be as low as possible. A remarkable improvement in SNR threshold is observed when frequency number is increased from $N=10$ to $N=20$. For $N$ larger than 20, the improvement in threshold grows slowly. This result reveals that the frequency number required for concerto to work is not too much, even for a quite large measurement range.

Remark 3: We observe that concerto outperforms EF and DCRT in estimation accuracy, reliability and computation complexity simultaneously. Meanwhile, an extremely large UMR can be guaranteed.

Moreover, the signal model of INSAR [8] is the same as the one in this letter and the phase noise follows an hypergeometric distribution which can be approximated as a Gaussian one [14]. Therefore, the proposed method can also be applied to phase unwrapping in INSAR besides its application in geodesy [1, 2, 9].

\section{Conclusion and future work}

Combination of a modified BW method and two optimal estimations for MFPU is presented in the letter. The key idea behind concerto is to exploit the modified BW for fast initial estimate and two optimal estimations for recovering the lost information caused by BW, thus maintaining the merit of both. As a result, concerto is highly attractive for its accurate and reliable distance estimation as well as extremely low complexity.

\section{Appendix}

\section{Appendix A. derivation of (19)}

We rewrite (18) as

$$
\begin{aligned}
& \max _{\hat{L}_{r}}\left(\sum_{i=0}^{N-1} e^{j\left(\frac{2 \pi f_{i} \hat{L}_{r}}{c}-\tilde{\phi}_{i}\right)}\right)\left(\sum_{k=0}^{N-1} e^{-j\left(\frac{2 \pi f_{k} \hat{L}_{r}}{c}-\tilde{\phi}_{k}\right)}\right) \\
= & \max _{\hat{L}_{r}} \operatorname{Re}\left\{\sum_{i=0}^{N-2} \sum_{k=i+1}^{N-1} e^{j\left(\frac{2 \pi\left(f_{i}-f_{k}\right) \hat{L}_{r}}{c}-\left(\tilde{\phi}_{i}-\tilde{\phi}_{k}\right)\right)}\right\}
\end{aligned}
$$

\footnotetext{
${ }^{5}$ For more information on the threshold effect, see $[15]$
} 


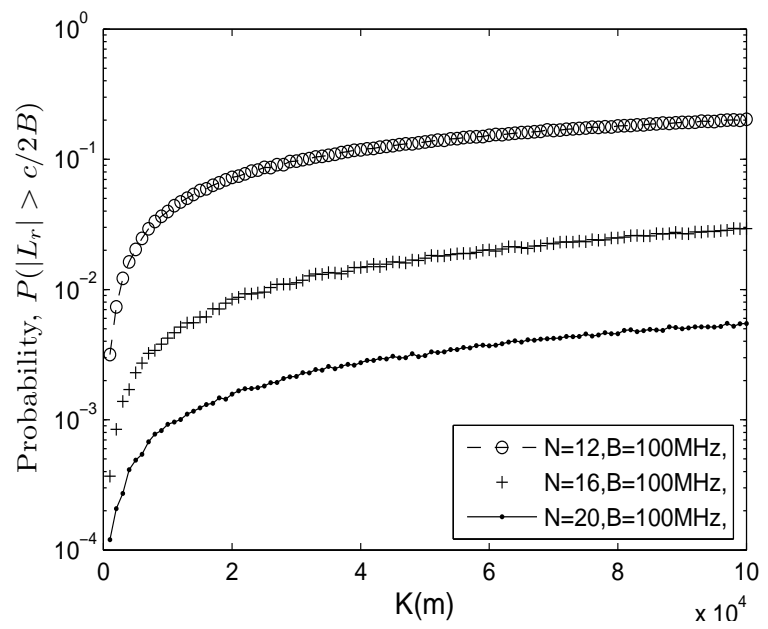

Figure 4: Probability $P\left(\left|L_{r}\right|>c / 2 B\right)$ versus $K$ under different $N$ with $\mathrm{SNR}=5 \mathrm{~dB}$.

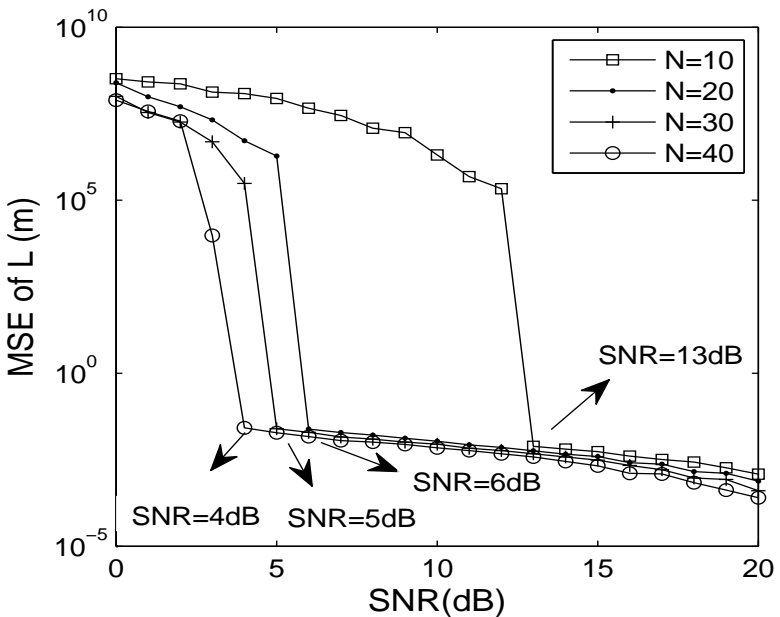

Figure 5: SNR threshold of concerto versus $N$ using the proposed frequency pattern with $K=100 \mathrm{~km}$ and $B=100 \mathrm{MHz}$.

Define

$$
\begin{aligned}
& \phi_{i, k}\left(\hat{L}_{r}\right)=\frac{2 \pi\left(f_{i}-f_{k}\right) \hat{L}_{r}}{c}-\left[\tilde{\phi}_{i}-\tilde{\phi}_{k}\right]_{2 \pi} \\
& =\frac{2 \pi\left(f_{i}-f_{k}\right) \hat{L}_{r}}{c}-\Delta \tilde{\phi}_{i, k} \\
& =\frac{2 \pi\left(f_{i}-f_{k}\right) \hat{L}_{r}}{c}-\left[\frac{2 \pi\left(f_{i}-f_{k}\right) L_{r}}{c}+\theta_{e}(i)-\theta_{e}(k)\right]_{2 \pi} \\
& =\frac{2 \pi\left(f_{i}-f_{k}\right) \hat{L}_{r}}{c}-\left(\frac{2 \pi\left(f_{i}-f_{k}\right) L_{r}}{c}+\theta_{e}(i)-\theta_{e}(k)\right)
\end{aligned}
$$


where $\Delta \tilde{\phi}_{i, k}=\left[\tilde{\phi}_{i}-\tilde{\phi}_{k}\right]_{2 \pi}$. The last equality holds since $\left|L_{r}\right|<\frac{c}{2 B}<\left|\frac{c}{2\left(f_{i}-f_{k}\right)}\right|$. According to (A.2), the "good" $\hat{L}_{r}$ must satisfy $\left|\phi_{i, k}\left(\hat{L}_{r}\right)\right| \ll 1$. Using a second-order approximation of Taylor series $f(x)=e^{j x}$ at $x_{0}=0$,

$$
\begin{aligned}
f(x) & \approx f\left(x_{0}\right)+\frac{f^{\prime}\left(x_{0}\right)}{1 !}\left(x-x_{0}\right)+\frac{f^{\prime \prime}\left(x_{0}\right)}{2 !}\left(x-x_{0}\right)^{2} \\
e^{j x} & \approx 1+j x-\frac{1}{2} x^{2}
\end{aligned}
$$

we have

$$
\operatorname{Re}\left\{\sum_{i=0}^{N-2} \sum_{k=i+1}^{N-1} e^{j \phi_{i, k}\left(\hat{L}_{r}\right)}\right\} \approx \sum_{i=0}^{N-2} \sum_{k=i+1}^{N-1}\left(1-\frac{1}{2}\left(\phi_{i, k}\left(\hat{L}_{r}\right)\right)^{2}\right)
$$

Thus, (A.1) is equivalent to

$$
\min _{\hat{L}_{r}} \sum_{i=0}^{N-2} \sum_{k=i+1}^{N-1}\left(\phi_{i, k}\left(\hat{L}_{r}\right)\right)^{2}
$$

The problem can be expressed as [12]

$$
\min _{\hat{L}_{r}} \sum_{i=0}^{N-2} \sum_{k=i+1}^{N-1}\left(\phi_{i, k}\left(\hat{L}_{r}\right)\right)^{2}=\min _{\hat{L}_{r}} \boldsymbol{\Phi}^{T}\left(\hat{L}_{r}\right) \mathbf{R}^{-1} \boldsymbol{\Phi}\left(\hat{L}_{r}\right)
$$

where $\boldsymbol{\Phi}\left(\hat{L}_{r}\right)=\left[\phi_{0,1}\left(\hat{L}_{r}\right), \phi_{0,2}\left(\hat{L}_{r}\right), \cdots \phi_{0, N-1}\left(\hat{L}_{r}\right)\right]^{T}$, and $\mathbf{R}^{-1}$ is defined in $(20)$.

The optimal solution $L_{r}^{*}$ obey

$$
\begin{gathered}
\left.\frac{\partial\left(\boldsymbol{\Phi}^{T}\left(\hat{L}_{r}\right) \mathbf{R}^{-1} \mathbf{\Phi}\left(\hat{L}_{r}\right)\right)}{\partial\left(\hat{L}_{r}\right)}\right|_{\hat{L}_{r}=L_{r}^{*}}=0 \\
\left(\partial\left[\boldsymbol{\Phi}\left(\hat{L}_{r}\right)\right] / \partial\left(\hat{L}_{r}\right)\right)^{T} \mathbf{R}^{-1} \boldsymbol{\Phi}\left(L_{r}^{*}\right)=0
\end{gathered}
$$

Since $\partial\left[\phi_{0, i}\left(\hat{L}_{r}\right)\right] / \partial\left(\hat{L}_{r}\right)=\frac{2 \pi}{c}\left(f_{0}-f_{i}\right)=\frac{2 \pi}{c} \sum_{k=1}^{i} \Delta f_{k}$, where $\Delta f_{k}=f_{k-1}-f_{k}$, and

$$
\frac{\partial\left[\mathbf{\Phi}\left(\hat{L}_{r}\right)\right]}{\partial\left(\hat{L}_{r}\right)}=\frac{2 \pi}{c}\left[\Delta f_{1}, \Delta f_{1}+\Delta f_{2}, \cdots \sum_{k=1}^{N-1} \Delta f_{k}\right]^{T}=\frac{2 \pi}{c} \boldsymbol{\Gamma} \Delta \mathbf{f}
$$

It follows that

$$
\begin{aligned}
\Delta \mathbf{f}^{T} \boldsymbol{\Gamma}^{T} \mathbf{R}^{-1} \boldsymbol{\Phi}\left(L_{r}^{*}\right) & =0 \\
\Delta \mathbf{f}^{T} \boldsymbol{\Gamma}^{T} \mathbf{R}^{-1}\left(\frac{2 \pi L_{r}^{*}}{c} \boldsymbol{\Gamma} \Delta \mathbf{f}-\boldsymbol{\Gamma} \Delta \tilde{\boldsymbol{\Phi}}\right) & =0
\end{aligned}
$$

Then

$$
L_{r}^{*}=\frac{c}{2 \pi} \frac{\Delta \mathbf{f}^{T} \boldsymbol{\Gamma}^{T} \mathbf{R}^{-1} \boldsymbol{\Gamma} \Delta \tilde{\boldsymbol{\Phi}}}{\Delta \mathbf{f}^{T} \boldsymbol{\Gamma}^{T} \mathbf{R}^{-1} \boldsymbol{\Gamma} \Delta \mathbf{f}}
$$

\section{Appendix B. Proof of (30)}

It is well known that the phase noise in (6) follows the wrapped normal distribution due to modulo $2 \pi$ operation[13]. So the estimation is usually not unbias and the CRB does not exist. But the noise can be approximated as normal distribution under the assumption of high SNR. 
Consider the signal $y(k)=A e^{j 2 \pi L / \lambda_{k}}+n(k)$, where $L$ is the parameter to be estimated and $n(k)$ is the complex Gaussian noise with zero-mean and variance $\mathbf{E}\left[n^{2}(k)\right]=\sigma_{n}^{2}$ and the signal-to-noise ratio (SNR) is defined as $\mathrm{SNR}=$ $A^{2} / \sigma_{n}^{2}$. The signal can be expressed as

$$
\begin{aligned}
y(k) & =A e^{j 2 \pi \frac{L}{\lambda_{k}}}+n(k) \\
& =A e^{j 2 \pi \frac{L}{\lambda_{k}}}\left(1+n(k) e^{-j 2 \pi \frac{L}{\lambda_{k}}} / A\right)
\end{aligned}
$$

Let $n^{\prime}(k)=n(k) e^{-j 2 \pi L / \lambda_{k}} / A=n_{R}^{\prime}(k)+n_{I}^{\prime}(k) j$, then $\mathbf{E}\left[n^{\prime 2}(k)\right]=\sigma_{n}^{2} / A^{2}$ and $\mathbf{E}\left[n_{I}^{\prime 2}(k)\right]=\sigma_{n}^{2} /\left(2 A^{2}\right)$. Therefore

$$
\begin{aligned}
y(k) & =A e^{j 2 \pi L / \lambda_{k}}\left(1+n_{R}^{\prime}(k)+n_{I}^{\prime}(k) j\right) \\
& =A \sqrt{\left(1+n_{R}^{\prime}(k)\right)^{2}+n_{I}^{\prime 2}(k)} e^{\left(j 2 \pi L / \lambda_{k}+\theta_{e}(k)\right)}
\end{aligned}
$$

where $\theta_{e}(k)$ is phase noise. At high SNR, the following approximation holds

$$
\begin{gathered}
\theta_{e}(k) \approx \tan \left(\theta_{e}(k)\right)=\frac{n_{I}^{\prime}(k)}{1+n_{R}^{\prime}(k)} \approx n_{I}^{\prime}(k) \\
\mathbf{E}\left[\theta_{e}^{2}(k)\right]=\sigma^{2} \approx \sigma_{n}^{2} /\left(2 A^{2}\right)
\end{gathered}
$$

Define $\mathbf{y}=[y(1), y(2), \cdots y(N)]$ and $\varphi=[\varphi(1), \varphi(2), \cdots \varphi(N)], \varphi(k)=\left[2 \pi L / \lambda_{k}+\theta_{e}(k)\right]_{2 \pi}$. It is clear that the original problem of estimating $L$ using $\varphi$, corrupted by noise of variance $\sigma_{n}^{2} /\left(2 A^{2}\right)$, is equivalent to estimating it from noisy signal $\mathbf{y}$ with noise variance $\sigma_{n}^{2}$. The Cramer-Rao bound of the equivalent problem is easily obtained as follows.

Let $a_{k}=\operatorname{Re}\{y(k)\}, b_{k}=\operatorname{Im}\{y(k)\}$. The probability distribution function is[16]

$$
\begin{array}{r}
f(\mathbf{y}, L)=\left(\frac{1}{\sqrt{\pi \sigma_{n}^{2}}}\right)^{2 N} \exp \left[-\frac{1}{\sigma_{n}^{2}} \sum_{k=1}^{N}\left(\left(a_{k}-A \cos \left(2 \pi L / \lambda_{k}\right)\right)^{2}\right.\right. \\
\left.\left.+\left(b_{k}-A \sin \left(2 \pi L / \lambda_{k}\right)\right)^{2}\right)\right]
\end{array}
$$

The entry of Fisher information matrix and the Cramer-Rao bound (CRB) for $L$ estimation in MFPU are given by

$$
\begin{aligned}
{[\mathbf{F}]_{L, L} } & =\mathbf{E}\left[\frac{\partial \log f(\mathbf{y}, L)}{\partial L} \frac{\partial \log f(\mathbf{y}, L)}{\partial L}\right] \\
& =\frac{8 A^{2} \pi^{2}}{\sigma_{n}^{2}} \sum_{k=1}^{N} \lambda_{k}^{-2} \\
\operatorname{CRB}(L) & =\left([\mathbf{F}]_{L, L}\right)^{-1}=\frac{\sigma^{2}}{4 \pi^{2}}\left(\sum_{k=1}^{N} \lambda_{k}^{-2}\right)^{-1}
\end{aligned}
$$

\section{References}

[1] B. Kusy, M. Maroti, G. Balogh, P. Volgyesi, J. Sallai, A. Nadas, A. Ledeczi, and L. Meertens, "Node density independent localization," in Proc. of 5th International Symposium on Information Processing in Sensor Networks (IPSN/SPOTS), Apr. 2006.

[2] C. Wang, Q. Yin, and H. Chen, "Robust Chinese remainder theorem ranging method based on dual-frequency measurements," IEEE Trans. Veh. Technol., vol. 60, pp. 4094-4099, 2011.

[3] X.-G. Xia and G. Wang, "Phase unwrapping and a robust Chinese remainder theorem," IEEE Signal Process. Lett., vol. 14, no. 4, pp. 247-250, Apr. 2007.

[4] X. W. Li and X.-G. Xia, "A fast robust Chinese remainder theorem based phase unwrapping algorithm," IEEE Signal Process. Lett., vol. 15, pp. 665-668, Oct. 2008.

[5] W. J. Wang and X.-G. Xia, "A closed-form robust Chinese remainder theorem and its performance analysis," IEEE Trans. Signal Process, vol. 58, pp. 5655-5666, Nov. 2010.

[6] X. Li and X.-G. Xia, "A new robust Chinese remainder theorem with improved performance in frequency estimation from undersampled waveforms," Signal Processing 117 (2015): 242-246. 
[7] Z. Huang and Z. Wan, "Range ambiguity resolution in multiple PRF pulse Doppler radars," in Proc. Int. Conf. Acoustics, Speech, Signal Processing (IEEE ICASSP), Dallas, TX, pp. 1786-1789, Apr. 1987.

[8] Z. H. Yuan, Y. k. Deng, F. Li et al., "Multichannel InSAR DEM reconstruction through improved closed-form robust Chinese remainder theorem," IEEE Geoscience and Remote Sensing Letters, vol. 10, pp. 1314-1318, Nov. 2013.

[9] W. C. Li, X. Z. Wang, X. M. Wang, and B. Moran, "Distance estimation using wrapped phase measurements in noise," IEEE Trans. Signal Process, vol. 61, pp. 1676-1688, 2013.

[10] K. Falaggis, D. P. Towers, C. E. Towers, "Method of excess fractions with application to absolute distance metrology: theoretical analysis," Appl. Opt, vol. 50, pp. 5484-5498, Oct. 2011.

[11] C. E. Towers, D. P. Towers, J. D. C. Jones, "Optimum frequency selection in multifrequency interferometry," Opt. Lett. vol. 28, pp. 887-889, Jun. 2003.

[12] Y. W. Wu, S. Rhodes and E. H. Satorius, "Direction of arrival estimation via extended phase interferometry," IEEE Trans. Aerosp. Electron. Syst, vol.31, pp. 375-380, Jan. 1995.

[13] Y. Q. Cheng, X. Z. Wang, T. Caelli, and B. Moran, "Tracking and localizing moving targets in the presence of phase measurement ambiguities," IEEE Trans. Signal Processing, vol. 59, pp. 3514-3525, Aug. 2011.

[14] S.N. Lahiri, A. Chatterjee and T. Maiti, "Normal Approximation to the Hypergeometric Distribution in Nonstandard Cases and a SubGaussian Berryesseen Theorem," J. Statistical Planning and Inference, vol. 137, no. 11, pp. 3570-3590, Nov. 2007.

[15] F. Athley, "Threshold region performance of maximum likelihood direction of arrival estimators," IEEE Trans. Signal Process. vol.53, pp. 1359-1373, Apr. 2005.

[16] D. C. Rife and R. R. Boorstyn, "Single-tone parameter estimation from discrete-time observations," IEEE Trans. Inform. Theory, vol. IT-20, pp. 591-598, Sept. 1974.

[17] L. Wei, W. Shao, W. D. Qi, J. H. Chen,"Peak-to-Peak search: Fast and accurate DOA estimation method for arbitrary nonuniform linear array," Electronics Letters, vol. 51, no. 25, pp. 2078-2080, 2015.

[18] L. Wei, W. D. Qi, D. X. Chen, P. Liu, E. Yuan, "Optimal sequencing of a set of positive numbers with the variance of the sequence's partial sums maximized," Optimization Letters,. vol. 7, no. 6, pp. 1071-1086, Aug. 2013

[19] C. Zhang, W. D. Qi, P. Liu, L. Wei, "Multipath cancellation by frequency diversity: a training-free and analytical approach to accurate RSS ranging in ground-deployed wireless sensor networks," Electronics Letters, vol. 50, no. 6, pp. 471-473, 2014. 